\title{
Chaos Theory and Transportation Systems: An Instructive Example
}

\author{
By
}

Christopher Frazier, Graduate Student Researcher, The University of Texas at Austin. 6.9 E. Cockrell Jr. Hall, Austin, TX 78712-1076, stanforth@mail.utexas.edu

Kara M. Kockelman, Clare Boothe Luce Assistant Professor of Civil Engineering The University of Texas at Austin, 6.9 E. Cockrell Jr. Hall, Austin, TX 78712-1076 kkockelm@mail.utexas.edu, Phone: 512-471-0210, FAX: 512-475-8744

(Corresponding Author)

The following paper is a pre-print and the final publication can be found in Transportation Research Record No.1897: 9-17, 2004.

Presented at the 83rd Annual Meeting of the Transportation Research Board, January 2004

\begin{abstract}
Chaos theory is used to analyze highly complex systems and thus may be useful for transportation applications. In this paper, a series of analyses find and exploit chaos are outlined, including time delays and embedding dimensions, Fourier power series, the correlation dimension, the largest Lyapunov exponent, and predictions. As an example, traffic flow data is analyzed and found to be chaotic, though it is shown that this could be the result of highfrequency noise. When used with a low-pass filter, predictions based on chaos theory are shown to have greater predictive power than a nonlinear least-squares method.
\end{abstract}

\section{INTRODUCTION}

Transportation systems are complex entities. Their current state and future evolution depend greatly on myriad properties of interacting, often highly variable physical and human elements. Proper representation of all dynamics in a model is complex: while certain relationships can be developed through analysis, incorporation of immeasurable quantities, such as laws and social codes, creates further complications. Faced with such challenges, chaotic data analysis appears a promising option for many transportation applications. A branch of nonlinear analysis, chaos theory is used to describe non-repeating systems that are too complex for traditional techniques ${ }^{1}$. Importantly, chaos theory allows one to distinguish between random, probabilistic, and deterministic systems.

On both theoretical and practical levels, there are three major benefits of chaos theory for system analysis:

- It applies to highly nonlinear systems.

- It naturally accounts for all important system dynamics.

- It uncovers system information and relationships without having to uncover the laws or equations of the underlying dynamics.

While it will not provide one with equations to develop a traditional model, it extracts valuable information involving all system dynamics for general application. Chaos theory seems naturally applicable to transportation systems, yet little convincing real-world evidence exists. To this end, this paper illustrates such an application, in the context of traffic flow data. 
This traffic example serves simply as a context for the framework, tools, and details of system investigation that this paper provides.

\section{LITERATURE REVIEW}

A large amount of chaos theory literature has been published in the last decade. The "discoverer" of chaos, Lorenz (1993) provides helpful, entry-level discussion. Casdagli et al. (1992) present an effective introduction to chaos theory and analysis techniques. Hilborn (2001) offers a broad yet detailed examination of chaos, while Argyris et al. (1994) provide more mathematically complex coverage. Abarbanel (1996) offers an excellent presentation of chaotic data analysis techniques.

In the context of transportation, much less has been published. Prigogine and Herman (1971) modeled traffic integrating statistical mechanics with individual choice behavior and showed it to exhibit a high degree of complexity. Disbro and Frame (1990) demonstrate how the theoretically derived, Gazis, Herman and Rothery (GHR) traffic model (Gazis, et. al., 1961) is highly chaotic, even when applied to small (eight-car) systems. Van Zuylen et al. (1999) discussed the implications of human behavior, chaos and unpredictability for urban and transportation planning and forecasting. Safanov et al. (2002) showed that chaotic behavior in traffic can due to the delays in human reaction. Nair et al. (2001) analyzed traffic flow data and characterized it as chaotic; their work relates most closely to the application pursued here and is elaborated under the Empirical Analysis section. And Weidlich (2000) demonstrated how random-utility-based models of relatively simple social behaviors produced chaotic behavior. This work offers a detailed application of chaos to traffic flow data. And, in order to apply chaos tools, one must first understand chaos theory.

\section{CHAOS THEORY AND DATA ANALYSIS Introduction to Chaos}

Essentially, chaos is a nonlinear behavior that exists between the realms of periodic and random. At first glance, some chaotic systems may appear to regular and periodic, whereas others will appear strictly random; in both cases closer examination topples these assumptions. Strictly speaking, chaotic systems are deterministic and, the exact system state can be written:

$$
\mathbf{X}(t)=(\mathbf{x}(t), \mathbf{x}(t-\tau), \mathbf{x}(t-2 \tau), \ldots, \mathbf{x}(t-(k-1) \tau)
$$

where $t$ is a scalar index for the data series and $\tau$ is the interval of observations. ${ }^{2}$ Let $\mathbf{F}$ :

$\mathfrak{R}^{k} \rightarrow \mathfrak{R}^{k}$ be the nonlinear function governing the system; then, the future state of the system at any time $t+\tau$ can be ascertained. However, as no real-world system is likely to be completely deterministic, a (relatively small) probabilistic component, $p(t)$, with mean zero is added to account for random effects ( $\mathrm{Lu}$ and Smith, 1991):

$$
\mathbf{x}(t+\tau)=\mathbf{F}(\mathbf{X}(t))+p(t) \text {. }
$$

The state of the system, $\mathbf{X}(t)$, is critical to knowing the progression of a system, and even a small change in it will radically alter the manner in which the system evolves. Thus, after a short interval, the system effectively becomes unpredictable. This effect is known as sensitivity to initial conditions and is a hallmark of chaotic systems.

Given a set of initial conditions, a dissipative ${ }^{3}$, nonrandom system eventually will settle onto a path called an attractor. Depending on the nature of the system, this attractor may be a point, a closed path, or, in the case of a chaotic system, a more complicated object. The attractor is the geometric representation of Eq. 1 for some time $t$ large enough to allow dissipation of transient effects accompanying system initiation. For Eq. 2's $k$-dimensional chaotic system, the 
attractor will be a non-intersecting, bounded path with infinite length, yet able to be encapsulated in a finite, $k$-dimensional volume. This attractor repeats its own geometric patterns infinitely. ${ }^{4}$

Knowing all this, assuming, or even hypothesizing, that a transportation system is chaotic invites questions. For example, is human behavior ultimately deterministic? Lorenz (1993) believes that such an assumption is most likely misguided. Yet the goal is to model human behavior, not reproduce it. In transportation systems, legal and social constraints may bound behavior, allowing modeler to more accurately predict human actions and system evolution. In addition, Eq. 2's random component allows one to incorporate variations in human behavior, under the guise of probability. ${ }^{5}$ Underlying all of this is the basic assumption that transportation systems are complex, and that the evolution of the systems is guided by non-random elements: there is order contained within them and chaos theory may potentially be used to model this.

\section{Embedding Dimensions}

A key component of chaotic data analysis is Takens' (1981) embedding theorem. Given Eq. 1's dynamical system, a scalar data measurement, $s(t)=h(\mathbf{x}(t)): \mathfrak{R}^{k} \rightarrow \mathfrak{R}$, dependent on the system's complete dynamics can be reconstructed into a $d$-dimensional vector series:

$$
\mathbf{y}(t)=[s(t), s(t+T), s(t+2 T), \ldots, s(t+(d-1) T)]
$$

where integer $m>0$ and $T=m \tau$ is called the (time) lag. Takens' theorem says that, if $d$ is large enough, the vector series $\mathbf{y}(t)$ reproduces many of the important dynamical characteristics of the original series, $\mathbf{x}(t)$ (Abarbanel 1996). Thus, one does not need the original vector series in order to analyze many of the system properties of the data series. A scalar function $s(t)$ describing the system is all that is necessary.

To apply this theorem effectively, good choices for the lag $T$ and embedding dimension $d$ are needed. Choice of $T$ should provide low correlation between adjacent elements in the embedded vector (so that the original data series is not mimicked) without being too long. One can use the first minimum of the average mutual information function:

$$
I(\tau)=\sum_{s(n), s(n+\tau)} \operatorname{Pr}(s(t), s(t+T)) \log _{2}\left[\frac{\operatorname{Pr}(s(t), s(t+T))}{\operatorname{Pr}(s(t)) P(s(t+T))}\right] .
$$

which measures the average amount of information (bits) shared by two measurements (Abarbanel 1996). For a data series, the probabilities come from a histogram of the data points and data point pairs.

To understand how to select a good embedding dimension, $d$, it is helpful to understand what is happening geometrically. As $d$ increases in the reconstruction of a data series, the chaotic attractor "unfolds". When completely unfolded, a sequential path from one $d$ dimensional point to the next will never cross itself. If $d$ is too small, various paths of the projected attractor will cross.

The method of false nearest-neighbors (for example, see Arbanel (1996)) recognizes that, where the attractor's paths appear to cross, two neighboring points actually will be far away in the sequential order of the true embedded data series. Using this idea, Cao (1997) proposed a method for determining a good embedding dimension. Let

$$
E 1(d)=\frac{E(d+1)}{E(d)}
$$

with 


$$
E(d)=\frac{1}{N-d T} \sum_{t=0}^{N-d T-1} \frac{\left\|\mathbf{y}_{d+1}(t)-\mathbf{y}_{d+1}^{N N}(t)\right\|}{\left\|\mathbf{y}_{d}(t)-\mathbf{y}_{d}^{N N}(t)\right\|}
$$

and

$$
\left\|\mathbf{y}_{d}(t)-\mathbf{y}_{d}^{N N}(t)\right\|=\max _{0 \leq j \leq d-1}\left|s(t+j T)-s^{N N}(t+j T)\right|,
$$

where $N$ is the length of the original data series, the subscript $d$ refers to the embedding dimension, and the superscript $N N$ means the nearest neighbor to the other vector as defined by the metric of Eq. 7. As $d$ gets large enough, $E 1(d)$ will tend to one. The appropriate embedding dimension is given by the value of $d$ where $E 1(d)$ stops changing. Practically, this means choosing the $d$ where $E 1(d)$ begins to "level out", near unity.

Cao (1997) also proposed a related method for determining whether or not the original data series is random. Using a different metric,

$$
E 2(d)=\frac{E^{*}(d+1)}{E^{*}(d)}
$$

where

$$
E^{*}(d)=\frac{1}{N-d T} \sum_{t=0}^{N-d T}\left|s(t+d T)-s^{N N}(t+d T)\right|,
$$

a random signal will have an $E 2(d)$ that is close to unity for all values of $d$, whereas a chaotic signal will have an $E 2(d)$ that is less than one for small values of $d$.

\section{Determining the Presence of Chaos}

In analyzing data, an important step is determining the presence of chaotic behavior. As with all methods outlined in this paper, there is some degree of interpretation involved, and so a positive result (for chaos) in one of these tests is no guarantee that chaos exists. What these tests will do is distinguish clearly periodic and/or random data from chaos. An example of the potentially misleading aspects of these tests is discussed in the example at the end of this paper.

One method to determine the presence of chaos uses the following Fourier power spectrum $^{6}$ :

$$
P(\omega)=\frac{1}{N^{2}}\left|\sum_{t=0}^{N-1} s(t)\left(e^{-i(2 \pi / N) t \omega}\right)\right|^{2}
$$

For periodic data, the power spectrum will spike at frequencies that characterize the system, and lie close to zero for all others. For chaotic data, the spectrum will be broadband and have a broad peak (Figure 1). Truly random noise should have a constant valued power spectrum; but in practice, it can be difficult to distinguish very noisy data from truly chaotic behavior using this method. 

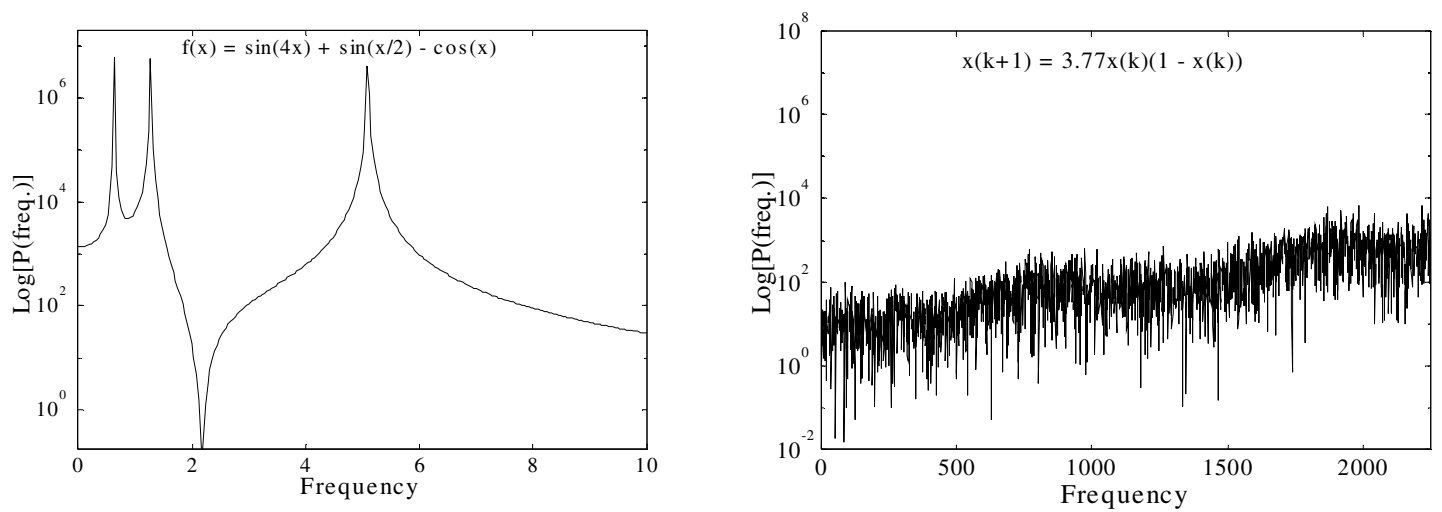

Figure 1. Fourier power spectra for periodic (left) and chaotic (right) logistic functions.

A more common technique to determine the presence of chaotic behavior is the largest Lyapunov exponent, which measures the divergence of nearby trajectories. As the system evolves, the sum of a series of attractor point values (in each dimension) will converge or diverge. Lyapunov exponents measure the rate of convergence/divergence in each dimension, and a chaotic system will exhibit trajectory divergence in at least one dimension. Thus, if the largest Lyapunov exponent exceeds zero, then the system is chaotic. To determine the largest Lyapunov exponent, the following function is used: ${ }^{7}$

$$
\lambda_{\max }=\frac{1}{N \Delta t} \sum_{t=0}^{N-1} \ln \left(\frac{\left|s(t+\Delta t)-s^{\prime}(t+\Delta t)\right|}{\left|s(t)-s^{\prime}(t)\right|}\right),
$$

where the primed and unprimed data points are distinct but close (i.e., within 10 or so time steps, [Hilborn 2001]). As $\Delta t$ increases, the value of the exponent will, theoretically, converge to its true value. In practice, however, due to a finite data sets and noise, the exponent can only be determined within a range of values.

Another indicator is a fractal dimension, which will be non-integer for chaotic systems. There are many types of fractal dimensions (Abarbanel 1996), but the most useful is the socalled correlation dimension. The following discussion is based on Hilborn (2001). If one takes a sphere of radius $R$ centered around a particular data point in embedded space, then the average number of data points contained in the sphere, not including the one at the center of the sphere, is given by:

$$
C(R)=\frac{1}{N(N-1)} \sum_{i=0}^{(N-d T-1)} \sum_{j=0, j \neq i}^{(N-d T-1)} \Theta(R-|\mathbf{y}(i)-\mathbf{y}(j)|)
$$

where $s(i)$ is the center of the sphere and $\Theta(x)$ is the Heavyside indicator function:

$$
\Theta(x)=\left\{\begin{array}{l}
0 \text { if } x<0 \\
1 \text { if } x \geq 0
\end{array} .\right.
$$

The correlation dimension assumes that as $R$ approaches zero, the rate at which $C(R)$ changes is given by the relation:

$$
C(R)=\lim _{R \rightarrow 0} k R^{D_{C}}
$$

or, solving for $D_{C}$ :

$$
D_{C}=\lim _{R \rightarrow 0} \frac{\log C(R)}{\log R} .
$$


Since the data set will not be continuous, $R$ cannot get too close to zero (there will be no points in the sphere). To handle this situation, one plots $C(R)$ versus $R$ and selects the apparently linear portion of the graph. The slope of this portion will approximate $D_{C}$. If $D_{C}$ is integer, then the attractor is a "normal" geometric object, like a point $\left(D_{C}=1\right)$ or a surface $\left(D_{C}=2\right)$; if it is noninteger, then the attractor is "strange" and the system exhibits chaos.

\section{Prediction}

As discussed previously, though chaos is fundamentally deterministic, it is unpredictable beyond short intervals. This section covers how short this interval will theoretically be and how accurate predictions can be made within that period. The method follows those discussed in Abarbanel (1997), Casdagli et al. (1997), and McNames (1998).

The (approximate) period limit on accurate predictions of a chaotic system is a function of the largest Lyapunov exponent (Abarbanel 1997):

$$
\Delta t_{\max }=\frac{1}{\lambda_{\max }} .
$$

To be chaotic, the largest Lyapunov exponent must exceed zero. If it exceeds one, the maximum length of an accurate prediction is less than the data series sampling frequency. Thus, only for systems with Lyapunov exponents between zero and one are chaotic predictions of any practical use. If the exponent is much less than one, long, accurate predictions are possible.

For prediction, one starts with the unfolded attractor in $d$-dimensional space and time lag $T$. Given an initial vector $\mathbf{y}\left(t_{0}\right)$, one selects the $k$ nearest trajectories (not points) on the attractor, and then the $k$ nearest points to $\mathbf{y}\left(t_{0}\right)$, one on each trajectory. An average of these trajectories is used to find the next point on the predicted trajectory, $\mathbf{y}\left(t_{0}+d T\right){ }^{8}$ The predicted point is then set as the new starting vector and the process is repeated.

This predictive technique offers the best model possible under the context of chaos theory. Since truly chaotic systems are infinitely complex and extremely sensitive to changes in system conditions, it is doubtful that the function $\mathbf{F}(\mathbf{x}(t))$ from Eq. 2, or even an approximation of it, could be extracted from data. This problem is compounded by the abstraction of the data through the use of embedding dimensions. Thus, searching for $\mathbf{F}(\mathbf{x}(t))$ is most likely a futile task. Fortunately, chaos theory rigorously exploits the fact that information is contained in the data and can be used for (short term) predictions.

\section{EMPIRICAL ANALYSIS}

Thus far, this paper has described key features of chaotic systems, the computations most helpful in assessing the presence of chaos, and methods for predictive chaos applications. This section describes an application to traffic counts, and illustrates how chaotic data analysis may mislead.

Similar to Nair et al.'s application (2001), we apply chaotic data analysis techniques to traffic flow measurements. The data were downloaded from the Freeway Performance Measurement Project (PeMS) run by the University of California, Berkeley. The data come from inductive-loop detectors embedded a section of Interstate 80 near Sacramento, California. The 30 -second vehicle counts have been summed across all lanes and aggregated to five-minute counts (in order to smooth higher-frequency fluctuations). The data was collected over a fiveweek interval from noon on April 7, 2003, noon to noon on May 12, 2003. All calculations were performed on the TSTool add-on program for Matlab. 
Figure 2 shows the total time series. Nair et al. (2001) noted that weekend and weekday traffic flow patterns are of a different flavor and recommended they be analyzed separately. The different flow patterns in our data seem to suggest this, but to be certain, we apply Cao's method (Eq. 8) for determining whether or not a data series is random. Eq. 4 suggests a time delay of 35, and Figure 3 plots the results of Cao's method. E2 $(d)$ remains around one for all values of $d$, suggesting that this is random data. However, from the plot of the data we see that this data exhibits substantial periodicity (on the order of both a day and a week), so we follow Nair et al.'s (2001) conclusion and focus only on a workweek, April 14 through 19.

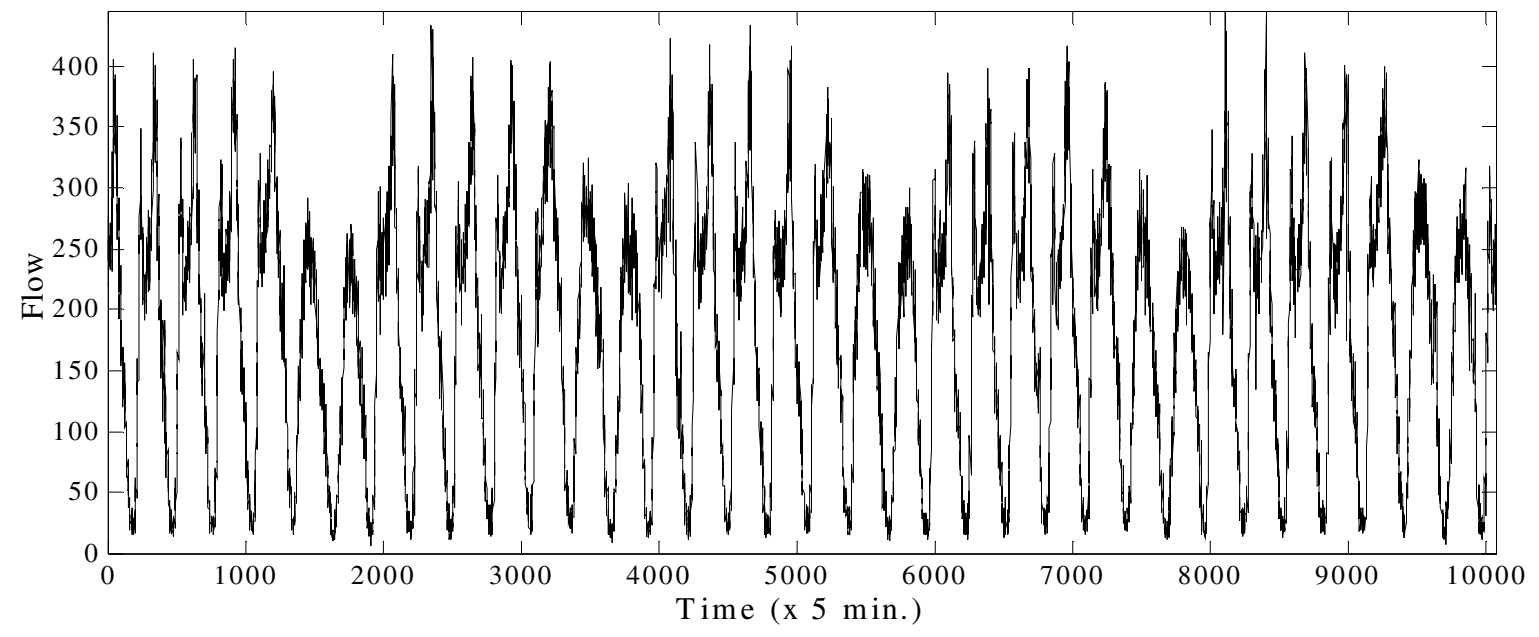

Figure 2. Time series of 5-minute traffic counts on Interstate 80 (noon on 4/7/2003 to noon on $5 / 12 / 2003)$.

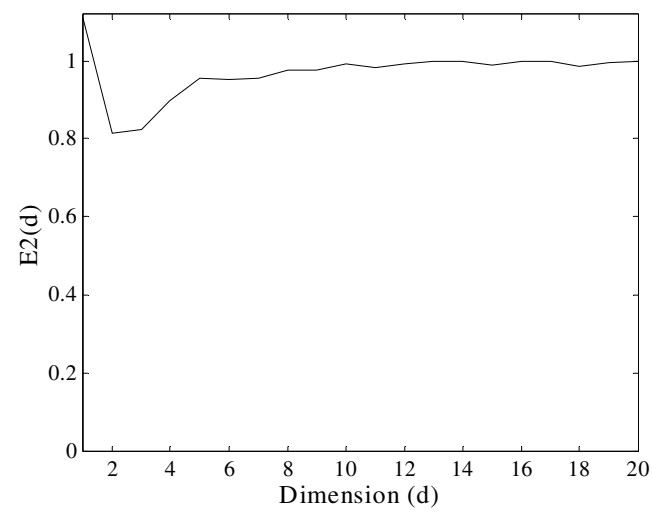

Figure 3. Plot of E2 $(d)$ for five-week traffic flow data

These five days of count data are plotted in Figure 4. For each day, there is a tight morning peak and a broader evening peak, corresponding to the morning and evening commutes. A strong periodic structure remains, and this will be important for the analysis.

Using the five days of weekday count data, one can first check for chaotic structure. Figures 5, 6, and 7 show the Fourier power spectrum, largest Lyapunov exponent, and correlation dimension, respectively. The broadband power spectrum and the positive Lyapunov exponent are characteristic of chaotic data. The linear part of the plot in Figure 7 has a slope of approximately 5.73 , indicating the presence of chaos. 


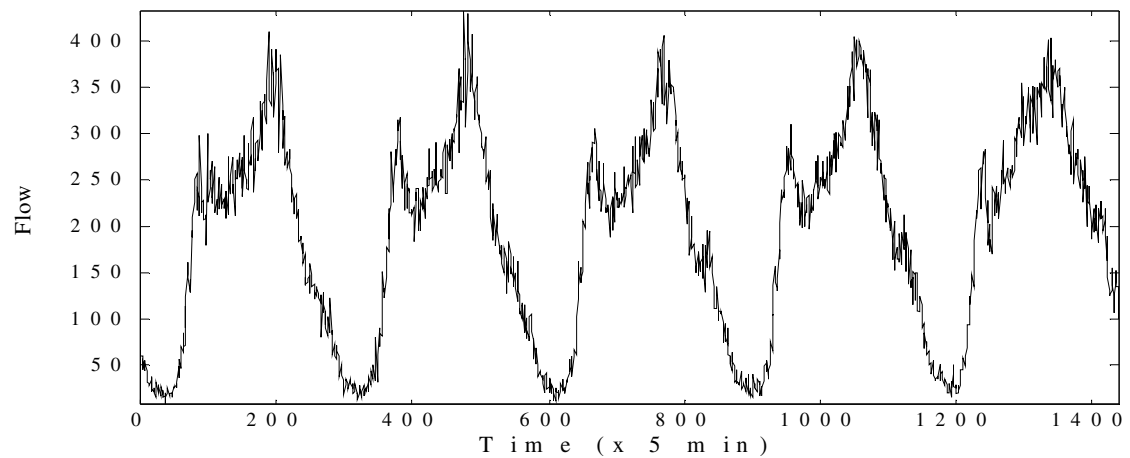

Figure 4. Time series of 5-minute traffic counts on Interstate 80 (midnight on 4/14/2003 through 11:55 pm on $4 / 18 / 2003$ )

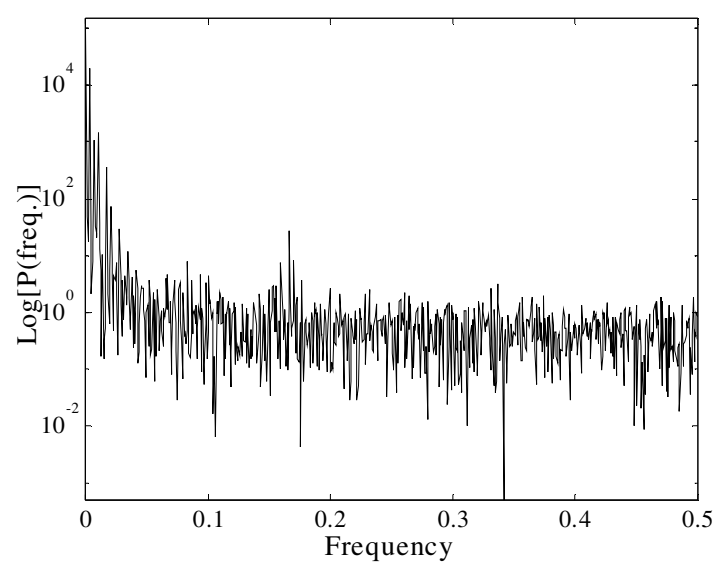

Figure 5. Fourier power spectrum for weekday traffic flow data.

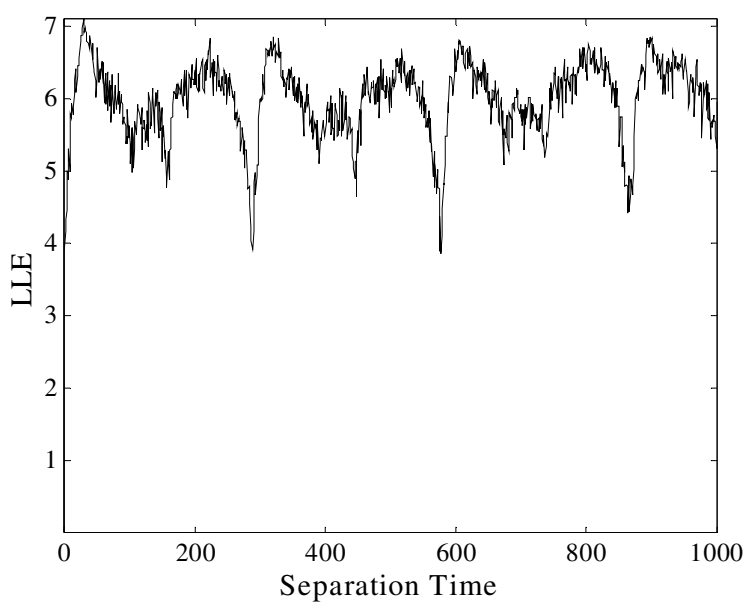

Figure 6. Largest Lyapunov exponent for weekday traffic flow data (Common values lie between 5 and 6.5, indicating chaos.) 


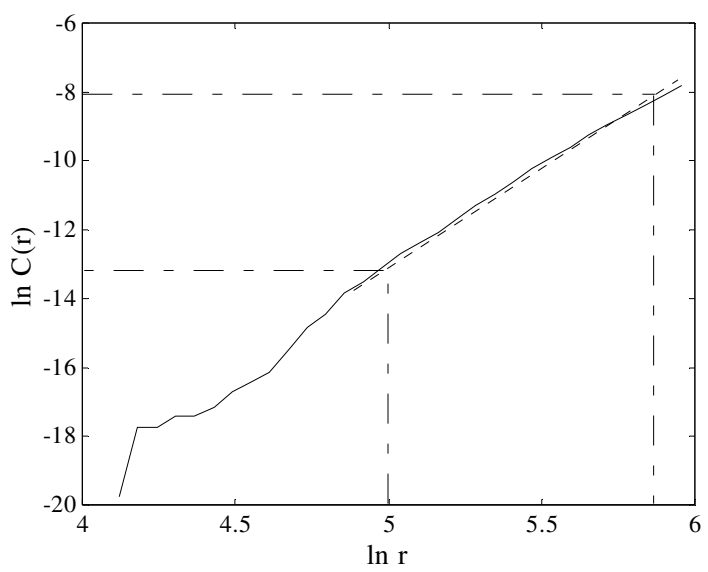

Figure 7. Correlation dimension for weekday traffic flow data (Linear portion, approximated by dotted line, has slope of about 5.73, indicating chaos.)

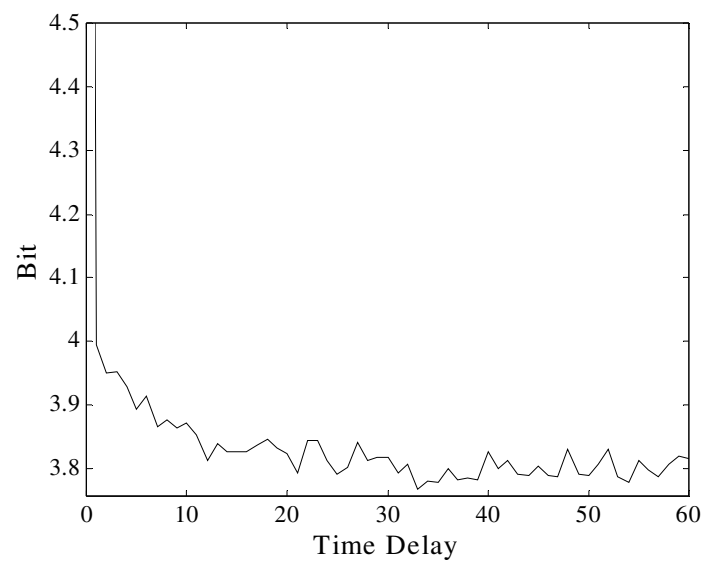

Figure 8. Mutual information function for weekday traffic flow data.

To facilitate computation of the embedding dimension parameters, Figure 8 shows the mutual information function. Though the first minimum occurs at a time delay of 3 (or 15 minutes), 12 (or 1 hour) is selected here, as it is more indicative of what the "true" first minimum of this function would be, if it was smooth.

Cao's methods (Eqs. 5-9) for determining $d$ and for determining whether data are random are plotted in Figure 9. The plot of E1 $(d)$ seems to level out at around 8, so this is selected this as the data set's embedding dimension. E2 $(d)$ is less than one for small $d$, suggesting that the series is not random.

Finally, the last two days of the week are predicted based on data from the first three days. Based on Eq. 16, the Lyapunov exponent suggests that the maximum window for accurate predictions is about 55 seconds, which is less than the sampling period of 5 minutes. Despite this, the predictions, shown in Figure 10, follow the actual data fairly accurately. Figure 11 provides a close view of the first few predictions, and the predictions appear much less accurate on the scale of the small oscillations. 

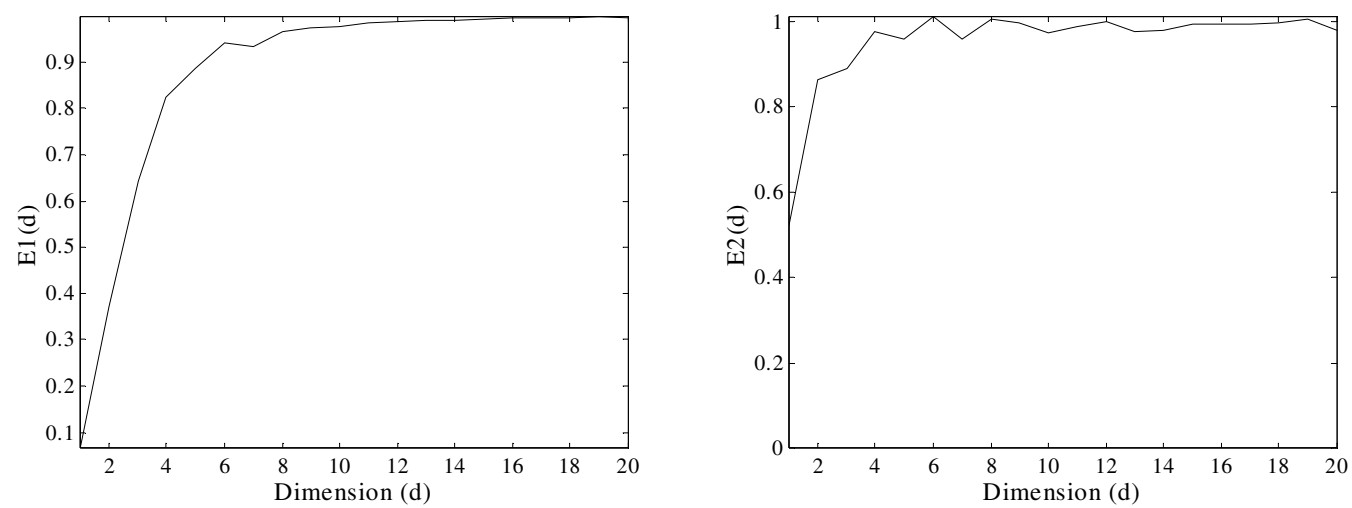

Figure 9. Plots of E1(d) (left) and E2(d) (right) for weekday traffic counts.

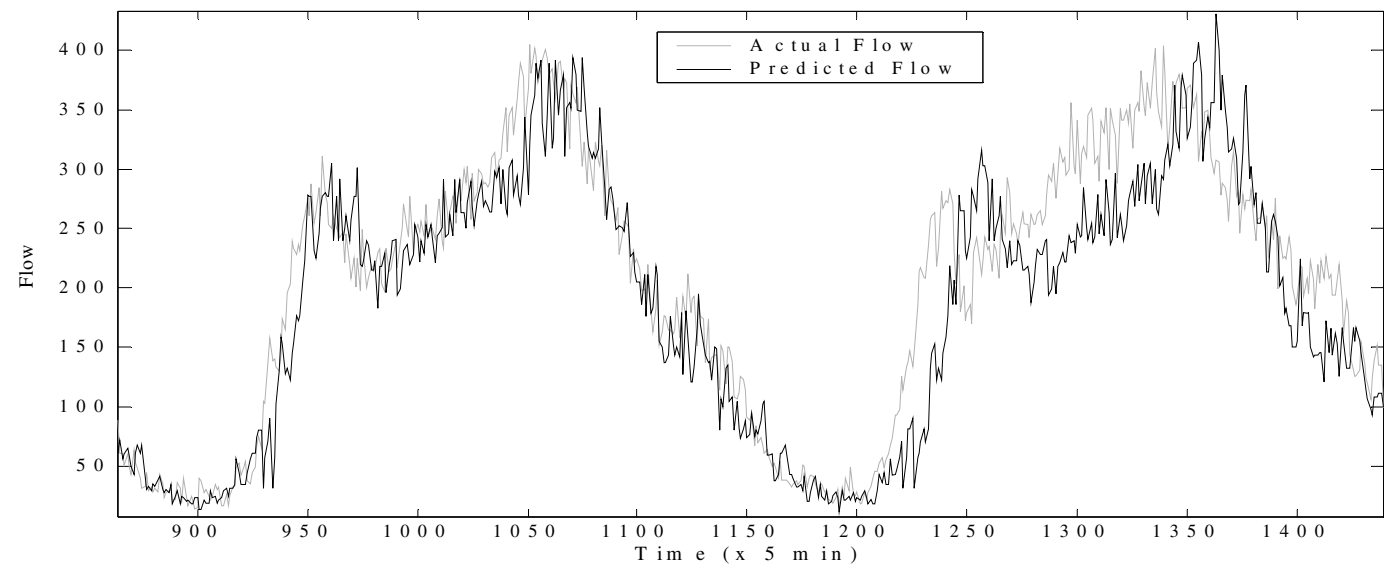

Figure 10. Comparison of actual and predicted Thursday and Friday traffic counts.

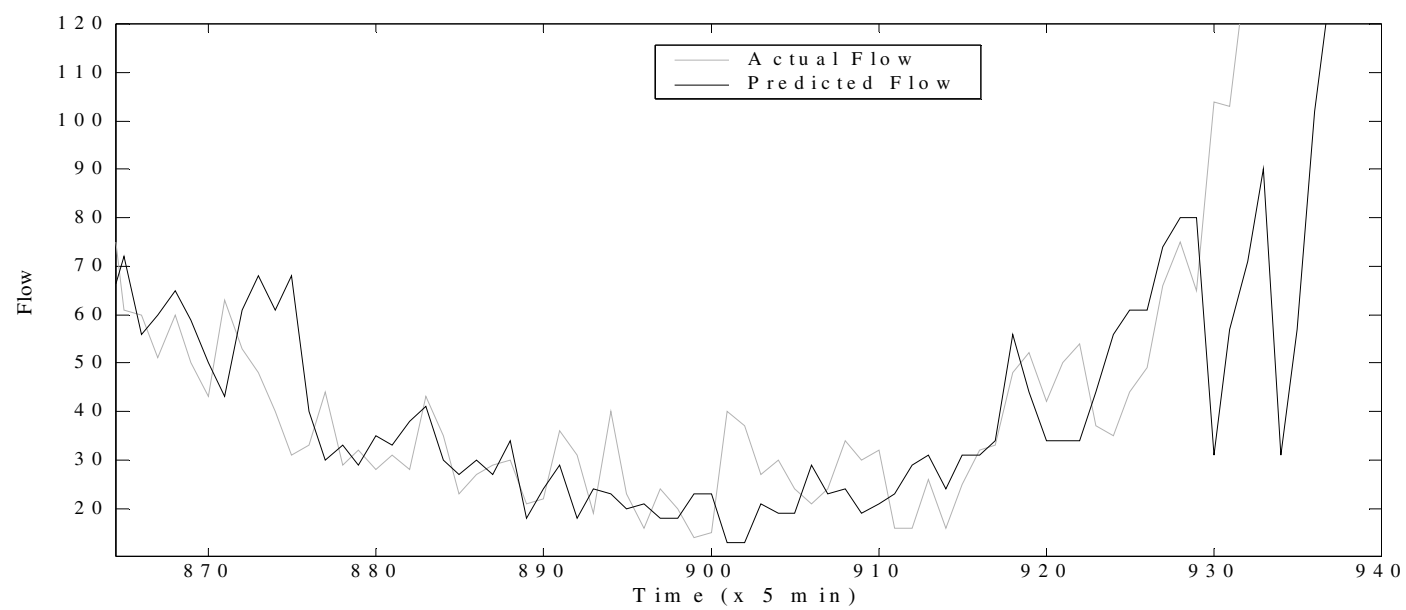

Figure 11. Close-up of actual and predicted counts. 
Why are these predictions, on a general level, so accurate? One possible reason is that the data's periodicity provides enough consistency and redundancy to produce good predictions. This answer provides a more important question: How do these data, which are so obviously periodic, appear to be chaotic? The key seems to lie in the high-frequency fluctuations that occur while the periodic path is traced. These could be random noise, or the result of chaotic processes. In the result of chaotic processes, the evidence of chaos in the analysis is logical. But if the data are truly random, these empirical results may suggest that a combination of noise and periodicity can mimic chaos.

To test this latter theory, a simple sinusoidal curve was created, and normally distributed noise was added (using a random number generator in Matlab, which itself is based on a semichaotic iterative function). Figure 12 illustrates two key results of the chaotic data analysis techniques for these data. They suggest the presence of chaos, and are very similar to results in the analysis of the weekday count data. However, since the "noise" is actually chaotic in origin (due to the mathematical basis for the "random" noise generator), one cannot determine whether the appearance of chaos is caused by the noise term's generation or the high frequency fluctuations interacting with the periodic macro-function. Yet one can conclude that, even if data are periodic on a large scale, certain high-frequency effects can, under analysis, lend the appearance of chaos.
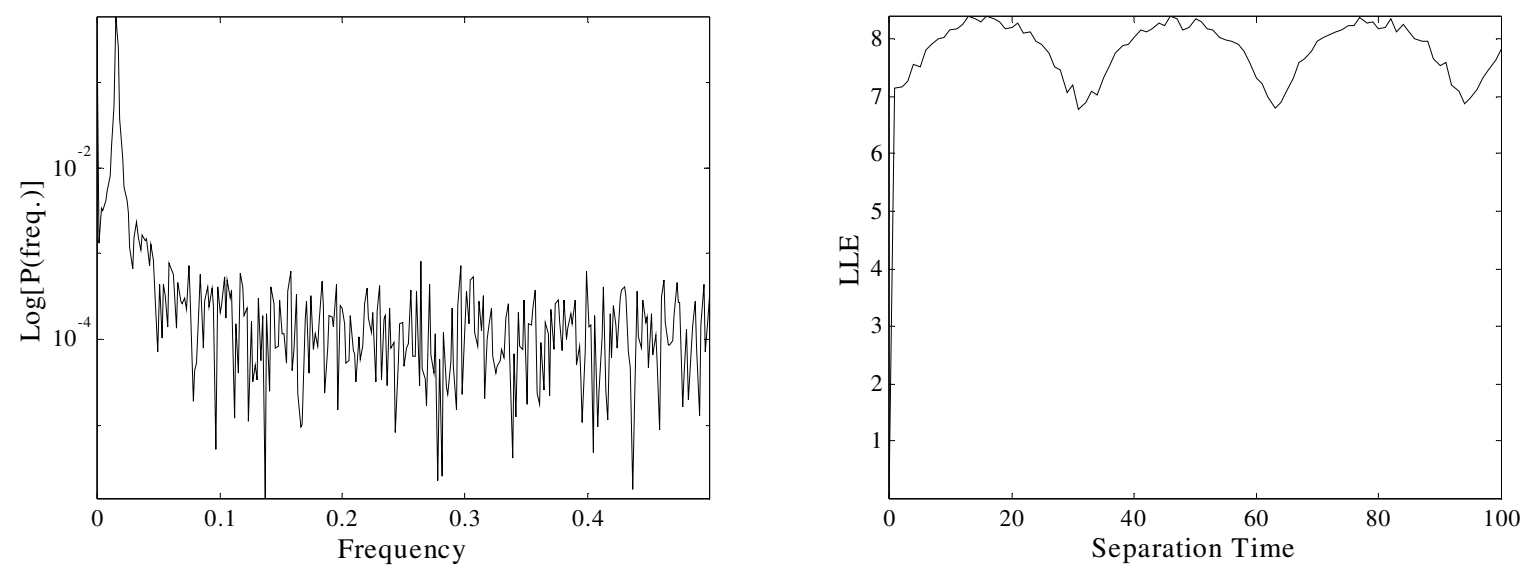

Figure 12. Fourier power spectrum of test function (left; spike at approximately 0.0152 corresponds to frequency of the base sin function); Largest Lyapunov exponent (right; lies between 6.75 and 8.5, indicating chaotic behavior).

However, if we accept this conclusion for our empirical example, the question becomes: what, if any, practical use does chaos theory have for this data? That is, the path of the high frequency fluctuations is not of much practical use (to modelers and system managers, for example), since they occur over very short, 5-minute intervals and are bound to a macro-level periodic structure that dictates the overall system path. ${ }^{9}$

To "create" an approximation of the macro-level structure using the actual predictions, a low-pass filter was applied to the predictions, removing the high-frequency fluctuations. ${ }^{10}$ The results of this are shown in Figure 13. In order to compare this technique to more traditional, statistical methods, a nonlinear least-squares routine was run using a combination of sin and cosine functions and was calibrated using the first three days of the week (to be consistent with the chaos-based method). The following function was obtained: 
Flow $=203.1+147.7 \sin (0.022 t-21.1)+29.7 \cos (0.042 t-22.4)+\varepsilon$.

Using this result, the subsequent two days have been plotted, along with the filtered chaos-based result, in Figure 13. A quantitative comparison using $\mathrm{R}^{2}$ values shows the chaos-based technique with filtering $\left(\mathrm{R}^{2}=0.949\right)$ is preferred to the least-squares method $\left(\mathrm{R}^{2}=0.841\right)$. Furthermore, as seen in Figure 13, the chaos-based method clearly captures the inter-day dynamics of the data, whereas the least-squares method barely registers them at all.

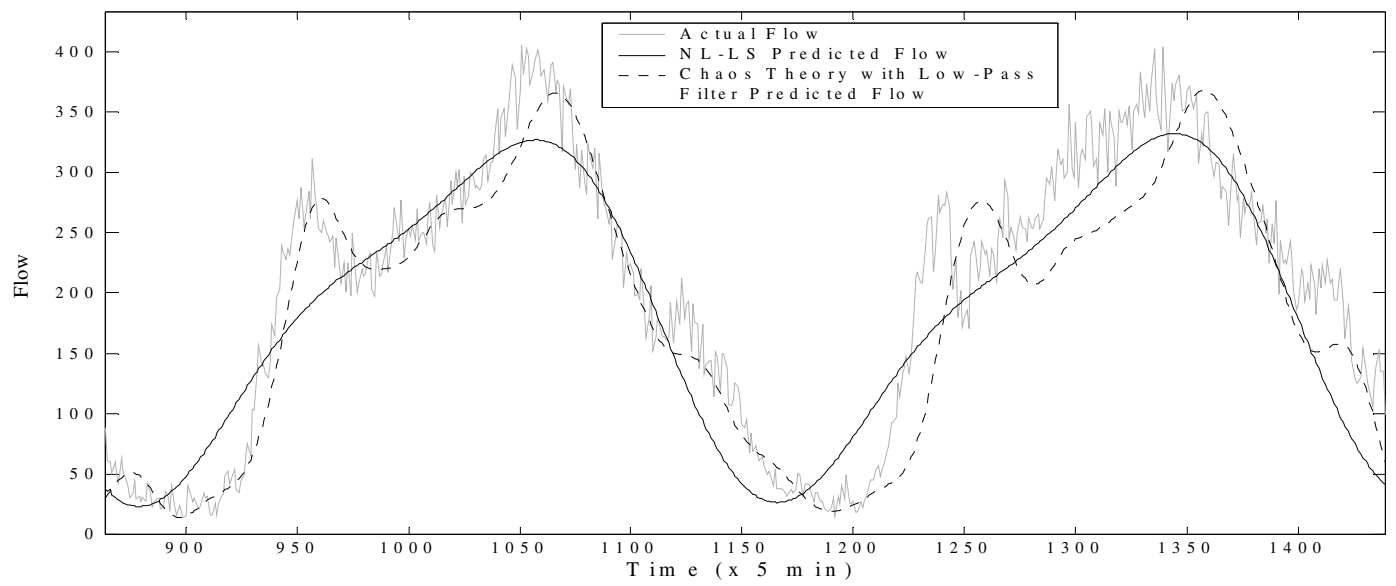

Figure 13. Comparison of Thursday and Friday traffic counts using chaos theory techniques with low-pass filtering and nonlinear least-squares methods.

\section{Conclusions}

This paper describes a series of analytic techniques for discerning and investigating chaotic dynamics and illustrates the application of such techniques to a series of traffic counts. It also shows that in certain cases, systems that are fairly periodic can analytically appear to be chaotic. Thus, care must be used in interpretation of results.

This paper was motivated by a hypothesis that transportation systems are often chaotic. The difficulty with this hypothesis is that chaos theory presumes system determinism. Since transportation systems involve humans, weather, and other possibly (probably?) random agents, such an assumption is not easy to justify. Thus, chaos theory may not well apply. However, if human behavior is controlled and directed through system laws and restrictions, then outcomes may be determined by system dynamics. And chaos theory may well apply.

Strong empirical evidence of chaos is needed for this theory to provide fruitful application in transportation systems modeling. Lack of good data is one concern: small measurement errors may be multiplied during analysis; and data that are not sufficiently extensive or are sampled at the wrong frequency will not accurately capture important system dynamics. Moreover, when selecting a scalar as the raw data source (e.g., traffic counts), logical and/or physical justification is necessary for that measurement's connection to and dependence on system properties; it must contain the information an analyst aims to extract. Furthermore, whether or not chaos exists, other dynamics may control the overall system evolution. This paper's traffic flow example is problematic because traffic counts are highly periodic. Indeed, while it seems rather obvious that the periodic portions of the data are deterministic, it is a much bigger step to say that the (chaotic) fluctuations are as well. 
Therefore, while chaos may exist on a small level, it may be neither discernable nor of (apparent) practical significance. This raises the issue of the usefulness of chaos theory to practitioners. And, if the analytical methods described in this paper do not provide explicit or explanatory models for the data, why use them? On a most basic level, the answer to this is simplicity. Though the methods described here may seem complex at first, they are actually quite easy to implement and are not data intensive; and they facilitate accurate results.

Furthermore, the assumptions of chaos theory - that the system is primarily deterministic and that the scalar measurement captures the dynamics of the system - in certain cases not be as restrictive as those of other models. ${ }^{11}$ Finally, if the system can be shown to be truly chaotic, then these techniques will probably the only manner through which useful information may be extracted from the system.

Chaotic data analysis, though unique, is not excessively complicated, and may serve as a powerful tool to transportation analysts. Many diverse areas could benefit, such as pavement analysis, flight logistics, and land use modeling. Using the techniques discussed in this paper, important properties of a system, such as its nature (periodic/repetitive, chaotic, or random) and its predictability, can be determined. Care must be taken, however, to justify the application of chaos theory to a system, so as not to extract information from a system that is clearly not (or could not be) chaotic. Though one should justify the application of chaos theory to a system (i.e. that it is chaotic), as the ambiguity of the source of chaos in the traffic flow example shows, this may not be necessary for good results. Moreover, and most importantly, while many system properties can be illuminated by chaotic data analysis, certain traditional properties, such as the system's defining equations, cannot be extracted through it. Even so, chaotic methods hold much promise for the analysis of many nonlinear transportation systems.

The methods discussed in this paper by no means cover all analytical techniques utilizing chaos theory. Many important topics have not been included for reasons of focus; these include bifurcation theory and universal scaling in chaotic systems (see Hilborn (2001)). One topic that may be highly useful in the context of transportation systems is symbolic data analysis. In this field, information is extracted from a system based on data abstractions and comparisons. Daw et al. (2001) provide an excellent survey of this topic. Additionally, there exist many freely available programs for analyzing chaotic data ${ }^{12}$. All are based on the premise that many systems harbor almost infinite complexity, and that complexity may be best addressed through application of chaos theory.

\section{Acknowledgements}

The authors wish to thank The University of Texas at Austin Research Internship program and the NSF CAREER Award program (Grant \# 9984541) for financial support of this research, and The University of California, Berkeley's Freeway Performance Measurement Project for providing the data. In addition, faculty and graduate students attending a presentation of an early form of this paper at an operations research colloquium at U.T. Austin provided many useful ideas and suggestions. 


\section{References}

Abarbanel, Henry D.I. Analysis of Observed Chaotic Data. Spinger-Verlag, New York. 1996. Argyris, John, Gunter Faust and Maria Haase. Texts on Computational Mechanics Volume VII: An Exploration of Chaos. North Holland, Amsterdam. 1994.

Casdagli, Martin, Deirdre des Jardins, Stephen Eubank, J. Doyne Farmer, John Gibson and James Theiler. "Nonlinear Modeling of Chaotic Time Series: Theory and Applications," in Applied Chaos, Jong Hyun Kim and John Stringer (Eds.). John Wiley and Sons, New York. 1992, pp. 335 - 380.

Cao, L. "Practical Method for Determining the Minimum Embedding Dimension of a Scalar Time Series," Physica D, Vol. 110. 1997, pp. 43 - 50.

Daw, C.S., C.E.A. Finny and E.R. Tracy. "A Review of Symbolic Analysis of Experimental Data." Submitted to Review of Scientific Instruments. 2001.

Disbro, John E. and Michael Frame. "Traffic Flow Theory and Chaotic Behavior," Transportation Research Record 1225, TRB, National Research Council, Washington D.C. 1989 , pp. 109 - 115.

Gazis, D.C., R. Herman and R.W. Rothery. "Nonlinear Follow-the-Leader Models for Traffic Flow," Operations Research 9, 1961, pp. 545 - 567.

Hilborn, Robert C. Chaos and Nonlinear Dynamics: An Introduction for Scientists and Engineers, 2nd Ed. Oxford University Press, Oxford.2001.

Lorenz, Edward N. The Essence of Chaos. University of Washington Press, Seattle. 1993.

McNames, J. "A Nearest Trajectory Strategy for Time-Series Prediction," Proc. of the International Workshop on Advanced Black-Box Techniques for Nonlinear Modeling. Katholieke Universiteit Leuven, Belgium. 1998, pp. 112 - 128.

Nair, Attoor Sanju, Jyh-Charn Liu, Laurence Rilett and Saurabh Gupta. "Non-Linear Analysis of Traffic Flow," 2001. http://translink.tamu.edu/docs/Research/LinearAnalysisTrafficFlow/chaos1.pdf. Accessed May 27, 2003.

Prigogine, Ilya, and Robert Herman. Kinetic Theory of Vehicular Traffic. Elsevier, New York. 1971.

Ramasubramanian, K., and M.S. Sriram. "A Comparative Study of Computation of Lyapunov Spectra with Different Algorithms," 2002. http://xxx.lanl.gov/PS_cache/chao-dyn/pdf/9909/9909029.pdf. Accessed May 27, 2003.

Safanov, L.A., E. Tomer, V.V. Strygin, Y. Ashkenazy and S. Havlin. "Delay-Induced Chaos with Multi-Fractal Attractor in a Traffic Flow Model," Europhysics Letters, Vol. 57. 2002, pp. $151-157$.

Sauer, Tim. "Reconstruction of Integrate-and-Fire Dynamics," in Fields Institute Communications, Volume 11: Nonlinear Dynamics and Time Series, Colleen D. Cutler and Daniel T. Kaplan (Eds.). American Mathematical Society, Providence. 1997, pp. 63 75.

Takens, F. "Detecting Strange Attractors in Turbulence," in Dynamic Systems and Turbulence, Warwick, 1980. Lecture Notes in Mathematics, D.A. Rand and L.S. Young (Eds.). Springer Verlag, Berlin. 1981, pp. 366 - 381.

Weidlich, Wolfgang. Sociodynamics: A Systematic Approach to Mathematical Modeling in the Social Sciences. Harwood Academic, Amsterdam. 2000. 
van Zuylen, Henk J., Marina S. van Geenhuizen and Peter Nijkamp. "(Un)predictability in Traffic and Transport Decision Making," Transportation Research Record 1685, TRB, National Research Council, Washington D.C. 1999, pp. 21-28.

\section{Endnotes}

${ }^{1}$ A more rigorous definition of chaos is provided later in this paper.

${ }^{2}$ Often, chaotic data series are time series, hence the use of the index $t$. This notational convention puts no restrictions on $t$, which represents any scalar index. Equation 1 also assumes all measurements are equally spaced by a distance $t$. This need not hold; see Sauer (1997) for an example.

${ }^{3}$ A dissipative system is essential one that requires a constant supply of energy to keep running. Thus, such a system will, over time, attempt to move towards an equilibrium in terms of energy (i.e. an attractor).

${ }^{4}$ Note that a system does not need to appear to be complex in order to be chaotic. An example of this is the logistic map: $x(k+1)=A x(k)(1-x(k))$, where $\mathrm{k}$ represents the $k$ th iteration and $A$ is a given constant.

5 Though it makes no explicit difference in the analysis, the application of chaos theory to transportation described in this paper assumes the use of the probabilistic term in Eq. 2. The justification for this is intuitive and comes from the assumption that most transportation problems will involve human, and thus some random (or probabilistic), elements. What is required for the application of chaos theory is that the deterministic part of the function dominate the probabilistic part.

${ }^{6}$ This is the discrete Fourier power spectrum, since we are assuming that this is applied to real, discretized (measured at distinct points) data. A different formula applies to continuous data.

${ }^{7}$ The entire Lyapunov exponent spectrum can be discerned, but this involves the complicated task of creating a local function approximation of the data series, and then finding the eigenvalues of the Jacobian of this approximation (see Arbanel (1997) and Ramasubramanian (1999)).

${ }^{8} \mathrm{~A}$ weighted average, giving greater value to trajectories closer to the initial vector, may also be used, though for our analysis this produced less accurate results (see McNames 1998).

${ }^{9}$ This is similar to the master-slave relationship of complex systems described in synergetics (e.g., Weidlich 2000). Essentially, quickly reacting (high-frequency) parts of a system will appear important on a micro-level, but on the order of the total system dynamics, it is the slow-moving, slow-reacting parts (i.e., the "order parameters") which control the system's evolution.

${ }^{10}$ The low-pass filter used employs multi-resolution analysis to remove multiple bands of high-frequency noise. Four levels $\left(4^{\text {th }}\right.$ scale) of noise were removed in this example. The filter is included in the TSTOOL package (see http://www.physik3.gwdg.de/tstool/ for more details).

${ }^{11}$ These two assumptions are probably the most important issues when considering the implementation of chaotic analysis to a system: The first allows for a justification of the use of the techniques (and may be more important than explaining why a system may be chaotic, since this latter question can be answered in the analysis), while the latter should be a guiding principle for selecting what type of scalar measurement to use for the analysis.

${ }^{12}$ For a listing of many of these programs, visit http://amath.colorado.edu/faculty/jdm/faq-\%5B5\%5D.html, which includes links to the vendors. 